\title{
The Assessment of Local Arterial Stiffness from Ultrasound Images
}

\author{
E Bianchini $^{1}$, C Giannarelli ${ }^{2}$, F Faita $^{1}$, K Raimo ${ }^{2}$, \\ V Gemignani $^{1}$, L Ghiadoni $^{2}, \mathrm{M} \mathrm{Demi}^{1,3}$ \\ ${ }^{1}$ CNR Institute of Clinical Physiology, Pisa, Italy \\ ${ }^{2}$ Department of Internal Medicine, University of Pisa, Pisa, Italy \\ ${ }^{3}$ Esaote SpA, Florence, Italy
}

\begin{abstract}
Local arterial stiffness of the superficial arteries can be evaluated by measuring the diameter change during the heart cycle from ultrasound data in conjunction with the local pulse pressure. In this work, such a system is introduced, and the obtained results on the common carotid artery are compared with those obtained by measuring the carotid to femoral pulse wave velocity $(P W V)$ which can be considered to be the "gold standard" technique for the evaluation of arterial stiffness. 14 healthy subjects and 14 hypertensive patients were involved in the study. Results show that a direct evaluation of local carotid stiffness, obtained by an appropriate video processing system, can discriminate between healthy and hypertensive patients as does the carotid-femoral PWV technique.
\end{abstract}

\section{Introduction}

Arterial stiffness is an independent predictor of cardiovascular morbidity and mortality and is thought to play a crucial role in the development of cardiovascular diseases. Currently it is widely used in interventional clinical studies to assess the effect of either nonpharmacological or pharmacological treatment on cardiovascular risk [1]. Some non-invasive techniques are available for the evaluation of the systemic, regional or local elasticity of the vessel walls $[2,3]$. In particular local arterial stiffness of superficial arteries, such as the carotid, can be estimated by measuring the diameter change during the heart cycle (stroke change in diameter or distension) from ultrasound data in conjunction with the local pulse pressure. In our lab a system with these capabilities was developed. The method evaluates the diameter of the artery by using a contour tracking technique applied to B-mode ultrasound images of a longitudinal section of the vessel. In this work the system is introduced, and the obtained results on the common carotid artery are compared with those obtained by measuring the carotid to femoral pulse wave velocity (PWV) by means of applanation tonometry. This measure is the "gold standard" technique for the evaluation of arterial stiffness [4].

\section{The system}

The developed system allows the automatic evaluation of the diameter change during the heart cycle. The method assesses the diameter of the artery by means of an edge detection technique applied to B-mode ultrasound sequences of the longitudinal section of the vessel. Figure 1 shows a typical B-Mode ultrasound image of the carotid artery and a schematic illustration of the main gray level discontinuities.

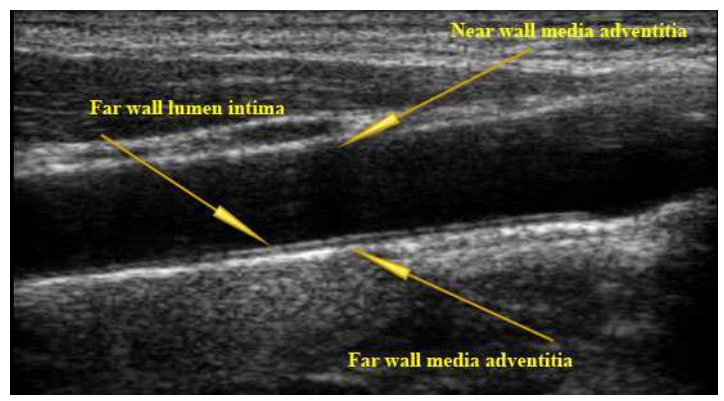

Figure 1. B-mode image of the longitudinal section of a carotid artery.

Meaningful edges can be mapped on the following interfaces: near wall media-adventitia (NMA), far wall lumen-intima (FLI) and far wall media-adventitia (FMA). For each image, the FLI and the FMA interfaces are automatically detected by using an algorithm [5] based on an edge operator (the First Order Absolute Moment [6]) and on a pattern recognition approach. The same algorithm is used to locate the NMA edge and the diameter is computed as the distance between the FMA and the NMA interfaces. Hence, for each frame of the sequence, the diameter and the far wall intima-media thickness (IMT) are available. 
Moreover, the data processing for the assessment of the distension includes some other computational stages:

$>$ The maximum (systolic) and minimum (diastolic) diameter values are identified for each cardiac cycle.

$>$ The stroke change in diameter is calculated for each cardiac cycle as the difference between the systolic and diastolic diameter values.

$>$ The mean distension value is computed as the average of the results obtained during the last 10 seconds.

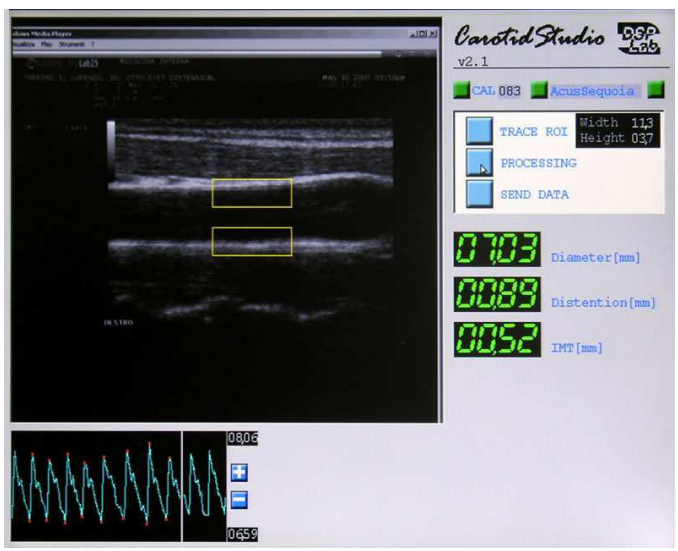

a)

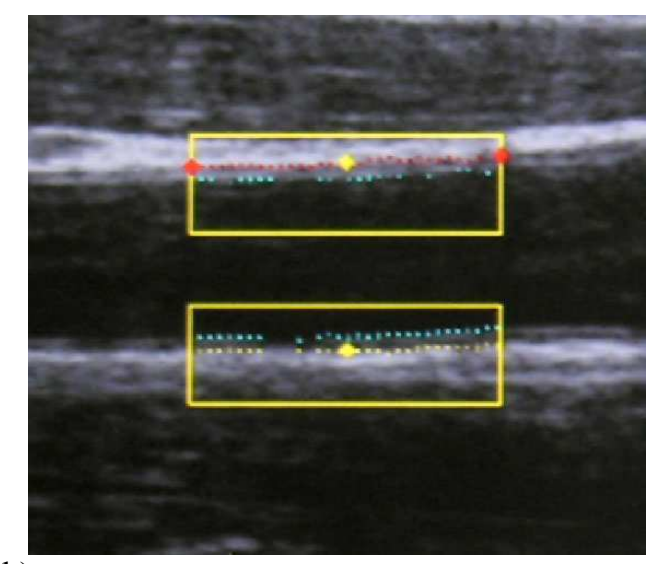

b)

Figure 2. GUI of the system (a) and particular of the interfaces detected by the algorithm (b).

The method was implemented on a stand-alone video processing system based on a DSP board which acquires the analog video signal from the ultrasound equipment and shows the results on a graphical user interface (GUI). The main component of the board is the Texas Instruments' TMS32C6415, a high performance DSP. A mouse and a keyboard are available to interact with the system and an ethernet link allows communication with a remote workstation. The algorithm was implemented in optimized $\mathrm{C}$ language and was integrated in the software of the board. The system works in realtime, with a frame rate of 25 frames/sec.

During the examination, once the medical operator has identified a region-of-interest (ROI), no other intervention is required; the instantaneous diameter waveform is automatically plotted on the GUI (Figure 2) and the found maxima and minima are highlighted with a mark in real-time. In this way the medical operator can take advantage of the visual feedback of the system and choose the most appropriate time interval within which to compute the distension with a simple click of the mouse : when the acquisition is done, the mean of the distension values and the mean of the diastolic diameter values of the last 10 seconds of the examination are shown. Moreover, the mean value of the far wall intima media thickness measurements is computed too. Hence from the same image sequence the system is able to furnish both elastic and structural parameters of the analysed vessel.

\section{Clinical experimentation}

Carotid to femoral pulse wave velocity (PWV) provides a regional evaluation of the vessel elasticity and can be considered to be the "gold standard" method for the assessment of arterial stiffness. Therefore, we compared the results obtained with this technique with those obtained by using our device for the carotid diameter evaluation. 14 healthy subjects (mean age $=25$ years) and 14 hypertensive patients (mean age $=41$ years) were involved in the study. Carotid pulse pressure (PP) and carotid to femoral PWV were carried out by applanation tonometry (SphygmoCor $\AA$ ), whereas artery diameter changes were evaluated with our system.

\subsection{Results}

The parameters of local elasticity [7] were evaluated. In particular compliance (C) and distensibility (Dist) were computed as:

$$
C=\frac{\Delta d}{P P} ; \quad \text { Dist }=\frac{C}{d_{D}}
$$

where $d_{D}$ represents the diastolic diameter, $\Delta d$ the stroke change in diameter and PP the local pulse pressure, respectively.

As shown in Figures $3 \mathrm{a}, 3 \mathrm{~b}$ and $3 \mathrm{c}$ the local stiffness parameters, which were obtained for healthy subjects $(\mathrm{C}$ $=0.01382 \pm 0.00277 \mathrm{~mm} / \mathrm{mmHg}$; Dist $=0.00207 \pm 0.00042$ $1 / \mathrm{mmHg})$ and for hypertensive subjects $(\mathrm{C}=$ $0.00842 \pm 0.00144 \mathrm{~mm} / \mathrm{mmHg}$; Dist $=0.00113 \pm 0.00021$ $1 / \mathrm{mmHg}$ ), clearly identifies the two different populations ( $\mathrm{p}<0.0001$ for both parameters) as well as the PWV data 
(healthy subjects: $\quad 5.333 \pm 0.427 \mathrm{~m} / \mathrm{s} ; \quad$ hypertensive subjects: $8.169 \pm 1.047 \mathrm{~m} / \mathrm{s} ; \mathrm{p}<0.0001)$.

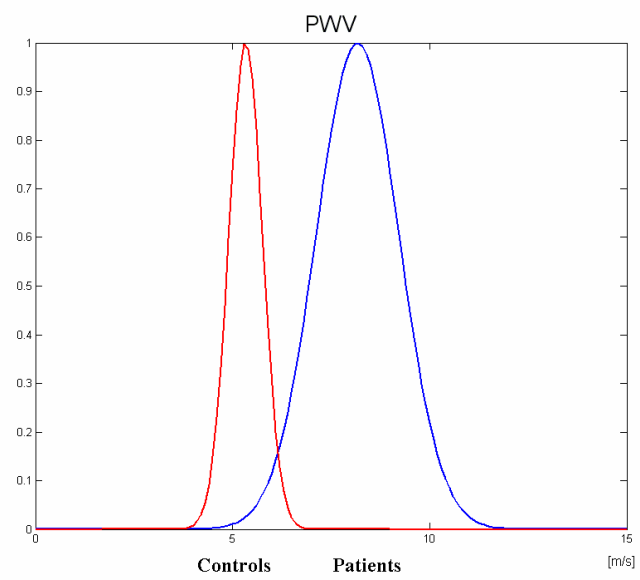

a)

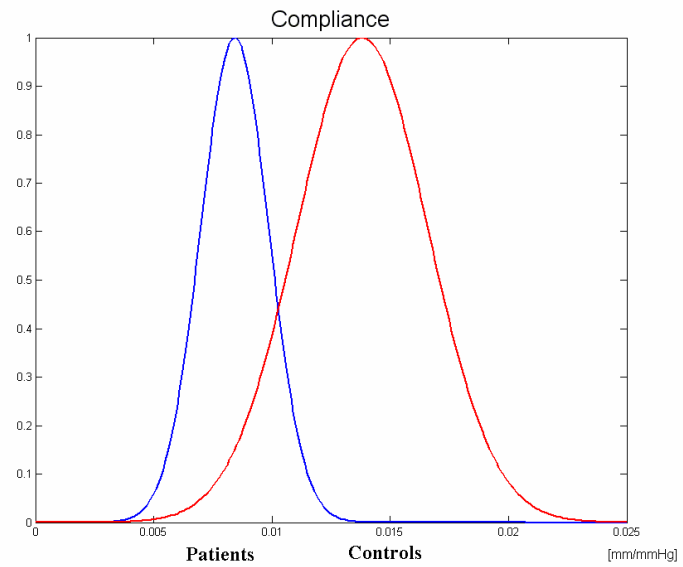

b)

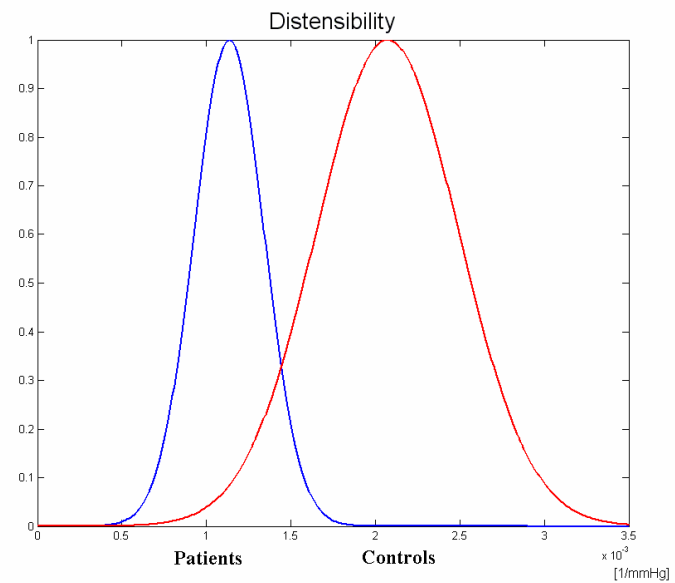

c)

Figure 3. Distributions of healthy and hypertensive subjects for carotid to femoral PWV (a), compliance (b) and distensibility (c).
Moreover, stroke change in lumen area $(\Delta \mathrm{A})$ and lumen area $(\mathrm{A})$ were evaluated from the diameter values, assuming the cross - section of the artery to be circular. Finally, the cross-sectional distensibility coefficient was calculated as

$$
D C=\frac{\Delta A}{\left(A_{D} * P P\right)}
$$

(where $A_{D}$ represents the diastolic lumen area) and it was converted, by using the Bramwell-Hill equation [8], into a parameter, CS, which has the same unit of measurement as the PWV and is computed as:

$$
C S=\frac{1}{\sqrt{D C^{*} \rho}}
$$

(where $\rho$ is the blood density). This local stiffness evaluation was also significantly $(p<0.0001)$ different in hypertensive subjects $(\mathrm{CS}=7.35 \pm 0.93 \mathrm{~m} / \mathrm{s})$ and controls $(\mathrm{CS}=5.76 \pm 0.74 \mathrm{~m} / \mathrm{s})$. Furthermore, carotid to femoral PWV was significantly $(\mathrm{p}<0.0001)$ correlated with CS ( $\mathrm{r}$ $=0.77)$ as shown in Figure 4.

Moreover, IMT values were also significantly $(\mathrm{p}<0.0001)$ different in hypertensive subjects (IMT $=$ $0.658 \pm 0.12 \mathrm{~mm}$ ) and controls (IMT $=0.542 \pm 0.084 \mathrm{~mm}$ ).

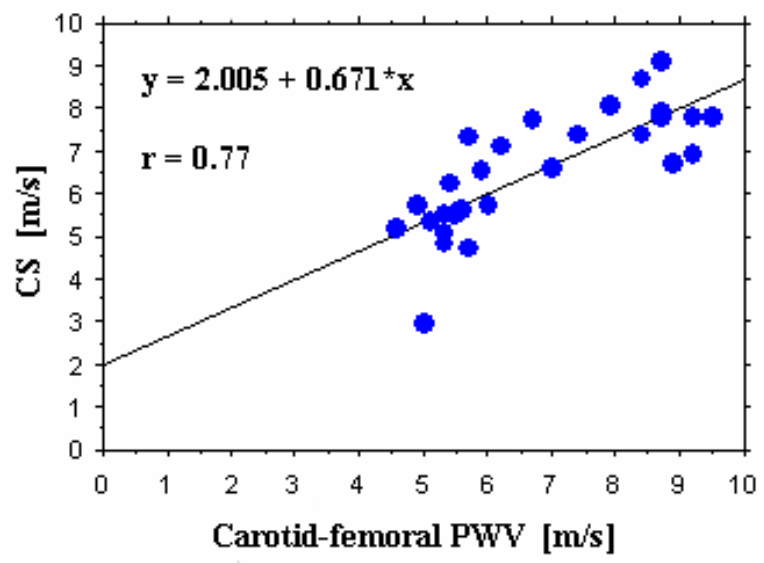

Figure 4. Regression line of CS versus carotid to femoral PWV.

\section{Discussion and conclusions}

Arterial stiffness is thought to have an important role in the development of cardiovascular diseases. Although, several non-invasive techniques are available for the assessment of systemic, regional or local arterial stiffness only the carotid to femoral PWV may be considered as the "gold standard" technique. Indeed, PWV, which provides a regional evaluation, is considered a simple, robust and reproducible technique and it has been used in epidemiological studies demonstrating its independent 
predictive value on cardiovascular events. However, some methodological limitations should be considered. In fact, the measurement of the distance between the sites of acquisition or of the transit time may be imprecise in some conditions (i.e. central obesity) and the femoral pressure waveform may be difficult to record accurately in subjects with metabolic syndrome, obesity, diabetes and peripheral artery disease [4].

Besides the PWV technique, the evaluation of stiffness in the carotid artery may be of great interest. So far, although several papers reported the independent predictive value of aortic stiffness on cardiovascular events, only few studies where parameters of local carotid elasticity were used are available in literature. These parameters can be obtained by measuring both the diameter change during the heart cycle from ultrasound data and the local pulse pressure. Hence, for this kind of evaluation both a system for measuring the instantaneous diameter and a method for the measurement of local pulse pressure are needed. Moreover, an ultrasound data processing system can additionally provide the automatic measure of carotid Intima-Media Thickness, thus allowing the evaluation of both elastic and structural parameters of a vessel from the same ultrasound signals which is considered optimal in the recent expert consensus document on arterial stiffness [4].

In this work we introduced a system for the automatic evaluation of local arterial elasticity parameters from ultrasound images. The device acquires the analog video signal from the ultrasound equipment and shows the results on a GUI in real-time. Once a ROI has been identified, no other intervention is required and the clinical operator can exploit the visual feedback of the system to hold the position of the probe and to choose the proper time to stop the acquisition of the data. Subsequently, we compared the results obtained by using this device on the carotid artery with those obtained from the carotid to femoral PWV. The preliminary results we derived by comparing the two methods, show that a direct evaluation of local carotid stiffness, carried out by an appropriate video processing system, can discriminate between healthy and hypertensive patients as does the carotid to femoral PWV technique.

Finally, we are aware that the results illustrated in this paper derived from a small study group. That is to say that a subsequent verification on a larger number of patients and controls is needed to better validate our device. Moreover, it is worth noting that the two techniques which were compared in this paper, should not be considered as alternative measurements: the carotid to femoral PWV provides a regional evaluation of arterial stiffness while our technique provides a local assessment of carotid elasticity. Therefore, the two techniques might be used together for a more accurate description of arterial stiffness.

\section{Acknowledgements}

This work was partially supported by the Regione Toscana (Tuscany, Italy) under the Grant IFC_Regione Toscana 2006.

\section{References}

[1] Laurent S, Kingwell B, Bank A, Weber M, StruijkerBoudier H. Clinical applications of arterial stiffness: therapeutics and pharmacology. American Journal of Hypertension 2002;15:453-458.

[2] Pannier BM, Avolio AP, Hoecks A, Mancia G, Takazawa K. Methods and devices for measuring arterial compliance in humans. American Journal of Hypertension 2002;15:743 -753 .

[3] Oliver JJ, Webb DJ. Non invasive assessment of arterial stiffness and risk of atherosclerotic events. Arteriosclerosis, Thrombosis and Vascular Biology Apr 2003; 23:554-556.

[4] Laurent S, Cockcroft J, Van Bortel L, Boutouyrie P, Giannattasio C, Hayoz D, Pannier B, Vlachopoulos C, Wilkinson I, Struijker-Boudier $\mathrm{H}$ on behalf of the European Network for Non-invasive Investigation of Large Arteries. Expert consensus document on arterial stiffness: methodological issues and clinical applications. European Heart Journal 2006; 27:2588-2605.

[5] Faita F, Gemignani V, Bianchini E, Giannarelli C, Demi M. Real-time measurement system for the evaluation of the Intima Media Thickness with a new edge detector. Proceedings of the $28^{\text {th }}$ Annual International Conference of the IEEE Engineering in Medicine an Biology Society 2006; 715-718.

[6] Demi M, Paterni M, Benassi A. The first absolute central moment in low level image processing. Computer Vision and Image Understanding 2000 Oct; 80(1):57-87.

[7] O'Rourke MF, Staessen JA, Vlachopoulos C, Duprez D, Plante GE. Clinical applications of arterial stiffness; definitions and reference values. American Journal of Hypertension 2002;15:426 - 444.

[8] Van Bortel LM, Duprez D, Starmans-Kool MJ, Safar ME, Giannattasio C, Cockcroft J, Kaiser DR, Thuileez C. Clinical applications of arterial stiffness, task force III: recommendations for users procedures. American Journal of Hypertension 2002;15:445 - 452.

Address for correspondence

Elisabetta Bianchini

Institute of Clinical Physiology

Via Moruzzi, 156124 Pisa, Italy

betta@ifc.cnr.it 